\title{
ON BOUNDEDLY METACOMPACT AND BOUNDEDLY PARACOMPACT SPACES
}

\author{
P. FLETCHER, R. A. MCCOY AND R. SLOVER ${ }^{1}$
}

ABstract. This paper introduces bounded paracompactness and relates the concept to compactness, paracompactness, and covering dimension.

1. Introduction. It is evident that every compact topological space is paracompact. Actually compactness implies a stronger property which we call bounded paracompactness. In this paper we study bounded paracompactness and a similar property, bounded metacompactness. We establish relationships between these properties and other well-known topological properties. In particular, bounded metacompactness is equivalent to bounded paracompactness in a collectionwise normal space. It follows from a theorem of $\mathrm{C}$. $\mathrm{H}$. Dowker [1, Theorem 3.5] that every paracompact topological space with finite covering dimension is boundedly paracompact. We show here that every locally infinite-dimensional boundedly paracompact space is compact and, in terms of dimension theory, we give a complete characterization of bounded paracompactness in hereditarily paracompact spaces.

Throughout this paper, dimension means covering dimension. For the sake of notational convenience, whenever we write $a=\left\{U_{\alpha}: \alpha \in A\right\}$ is a collection of sets indexed by a set $A$, it is understood that if $\alpha \neq \beta$ and $U_{\alpha}=U_{\beta}$ then $U_{\alpha}=U_{\beta}=\varnothing$.

\section{Preliminary results.}

Definition 1. Let $X$ be a topological space, let $n$ be a positive integer, and let $\mathfrak{e}$ be a collection of subsets of $X$. Then $\mathfrak{e}$ is point finite of order $n$ provided that each $x \in X$ is in at most $n$ members of $\mathfrak{C}$, and $\mathcal{C}$ is locally finite of order $n$ provided that each $x \in X$ has a neighborhood which intersects at most $n$ members of $\mathcal{C}$.

Presented to the Society, January 25, 1970; received by the editors July 22, 1969. A MS Subject Classification. Primary 5450, 5470; Secondary 5452.

Key Words and Phrases. Paracompact, boundedly paracompact, metacompact, boundedly metacompact, point finite cover, locally finite cover, collectionwise normal, hereditarily paracompact, finite-dimensional, infinite-dimensional, $n$-discrete collection, compact.

1 The authors thank Professor J. C. Smith for several valuable technical discussions as well as for his lectures on "Shrinkable Open Covers." 
Definition 2. A topological space $X$ is boundedly metacompact (boundedly paracompact) provided that if $\mathfrak{e}$ is an open cover of $X$, then there is a positive integer $n$ such that $\mathcal{C}$ has a point finite (locally finite) open refinement of order $n$.

Definition 3. Let $X$ be a topological space and let $n$ be a positive integer. Then $X$ is n-boundedly metacompact (n-boundedly paracompact) provided that $n$ is the least positive integer such that if $\mathcal{C}$ is an open cover of $X$, then $\mathcal{e}$ has a point finite (locally finite) open refinement of order $n$.

We begin by giving several theorems which are analogous to standard theorems concerning paracompactness. The proof of the first theorem follows from simple modifications of the corresponding theorem for metacompact and paracompact spaces. The second theorem resembles the widely known result of E. Michael [4, Lemma 1 and Theorem 1].

TheOREM 1. Let $n$ be a positive integer. The following properties are weakly hereditary:

(i) bounded metacompactness,

(ii) n-bounded metacompactness,

(iii) bounded paracompactness,

(iv) n-bounded paracompactness.

Definition 4 . Let $X$ be a topological space and let $n$ be a positive integer. A family $\mathcal{C}$ of subsets of $X$ is $n$-discrete if and only if it is the union of $n$ discrete subfamilies.

TheOREM 2. Let $X$ be a regular topological space. The following statements are equivalent:

(i) $X$ is boundedly paracompact.

(ii) If $\mathcal{C}$ is an open cover of $X$, there is a positive integer $n$ such that e has a locally finite closed refinement of order $n$.

(iii) If $\mathfrak{e}$ is an open cover of $X$, there is a positive integer $n$ such that $\mathfrak{e}$ has a locally finite refinement of order $n$.

(iv) If $\mathfrak{C}$ is an open cover of $X$, there is a positive integer $n$ such that e has an open n-discrete refinement.

Proof. The proof of the equivalence of (i), (ii), and (iii) follows with obvious minor changes from J. Kelley's presentation of the corresponding theorem for paracompact spaces [3, p. 156]. Also it is evident that (iv) implies (i) so that by the above remarks it is sufficient to show that (ii) implies (iv). Let $\mathfrak{e}$ be an open cover of $X$, let $\Re=\left\{R_{\alpha}: \alpha \in A\right\}$ be a locally finite closed refinement of $\mathcal{C}$ of order $n$ and, for each positive integer $i$ not greater than $n$, let 


$$
R_{i}=\left\{\bigcap_{k=1}^{i} R_{\alpha_{k}}-\bigcup_{\beta \neq \alpha_{k}} R_{\beta}: \alpha_{k}, \beta \in A(1 \leqq k \leqq i)\right\} .
$$

Note that $\bigcup_{i=1}^{n} R_{i}$ is a cover of $X$. We show first that $R_{n}$ is a discrete collection. Let $x \in X$. Then there exists a positive integer $i, 1 \leqq i \leqq n$, such that $x \in \bigcap_{\boldsymbol{k}=1}^{i} R_{\alpha_{k}}-\bigcup_{\beta \neq \alpha_{k}} R_{\beta} \quad(1 \leqq k \leqq i)$. Let $V=X-\bigcup_{\beta \neq \alpha_{k}} R_{\beta}$ $(1 \leqq k \leqq i)$. Then $V$ is an open set containing $x$. Let $Q \in \mathbb{R}_{n}$ such that $Q \neq \bigcap_{k=1}^{i} R_{\alpha_{k}}-\bigcup_{\beta \neq \alpha_{k}} R_{\beta}(1 \leqq k \leqq i)$. Then $Q$ is of the form $\bigcap_{k=1}^{n} R_{\gamma_{k}}$ $-\bigcup_{\beta \neq \gamma_{k}} R_{\beta}(1 \leqq k \leqq n)$ so that there exists a positive integer $j, 1 \leqq j \leqq n$, such that $R_{\gamma_{j}} \neq R_{\alpha_{k}}$ for any $k, 1 \leqq k \leqq i$. Hence $V \cap Q=\varnothing$ so that $R_{n}$ is discrete.

Since $X$ is paracompact, there is an open discrete collection $\mathfrak{R}_{n}^{\prime}$ such that each member of $R_{n}$ is contained in some member of $R_{n}^{\prime}$ and each member of $\boldsymbol{R}_{n}^{\prime}$ is contained in some member of $\mathcal{C}$. Let $\mathbb{R}_{n-1}^{\prime \prime}$ $=\left\{R-\cup R_{n}^{\prime}: R \in \mathbb{R}_{n-1}\right\}$. We show that $\mathcal{R}_{n-1}^{\prime \prime}$ is a discrete collection. Let $x \in X$ and suppose that $x \in \bigcap_{k=1}^{i} R_{\alpha_{k}}-\bigcup_{\beta \neq \alpha_{k}} R_{\beta}(1 \leqq k \leqq i)$ where $1 \leqq i \leqq n-1$. Then, as in the same manner as argued above, there is an open set $U$ containing $x$ which intersects at most one member of $\mathfrak{R}_{n-1}$. Hence $U$ intersects at most one member of $\mathbb{R}_{n-1}^{\prime \prime}$. If $x \in \cup \mathbb{R}_{n}$ $\subset \mathrm{U}{R_{n}^{\prime}}_{n}$, then $U \mathcal{R}_{n}^{\prime}$ is an open set containing $x$ which does not intersect any member of $\mathbb{R}_{n-1}^{\prime \prime}$. Thus $\mathbb{R}_{n=1}^{\prime \prime}$ is discrete, so that there exists an open discrete collection $R_{n-1}^{\prime}$ such that each member of $\mathbb{R}_{n-1}^{\prime \prime}$ is contained in some member of ${R_{n-1}^{\prime}}^{\prime}$ and each member of $\mathbb{R}_{n-1}^{\prime}$ is contained in some member of $\mathfrak{C}$.

As above we can define discrete open collections $\mathbb{R}_{1}^{\prime}, \mathfrak{R}_{2}^{\prime}, \cdots, \mathfrak{R}_{n}^{\prime}$ so that $\mathrm{U}_{i=1}^{n} \mathscr{R}_{i}^{\prime}$ covers $X$ and is an open $n$-discrete refinement of $\mathfrak{C}$.

THEOREM 3. Every boundedly metacompact collectionwise normal Hausdorff space is boundedly paracompact.

Proof. Let $X$ be a boundedly metacompact collectionwise normal Hausdorff space and let $u$ be an open cover of $X$. There is a positive integer $n$ such that $u$ has a point finite refinement $\mathcal{u}^{*}=\left\{U_{\alpha}: \alpha \in A\right\}$ of order $n$. Since $X$ is metacompact and collectionwise normal, $X$ is paracompact. Consequently, there is a locally finite open refinement $\mathcal{H}=\left\{H_{\beta}: \beta \in B\right\}$ of $\mathcal{U}^{*}$. Let $<$ be a well order on $A$ and for each $\beta \in B$ let $f(\beta)$ be the least $\alpha \in A$ such that $H_{\beta} \subset U_{\alpha}$. For each $\alpha \in A$ let $H_{\alpha}^{*}=\bigcup\left\{H_{\beta}: f(\beta)=\alpha\right\}$ and let $\mathfrak{H}^{*}=\left\{H_{\alpha}^{*}: \alpha \in A\right\}$. Then $\mathfrak{H}^{*}$ is a locally finite open refinement of $\mathcal{u}^{*}$ such that for each $\alpha \in A, H_{\alpha}^{*} \subset U_{\alpha}$. Let $\mathfrak{F}^{*}=\left\{F_{\alpha}: \alpha \in A\right\}$ be a closed shrinking of $\mathcal{F}^{*}$. Since $(X, \mathfrak{J})$ is normal, for each $\alpha \in A$ there is an open set $V_{\alpha}$ so that $F_{\alpha} \subset V_{\alpha} \subset \bar{V}_{\alpha} \subset H_{\alpha}^{*}$. Let $V=\left\{V_{\alpha}: \alpha \in A\right\}$. Then $v$ is an open locally finite refinement of $\mathcal{u}^{*}$ such that for each $\alpha \in A, V_{\alpha} \subset U_{\alpha}$. 
Let $x \in X$. Let $U=X-U\left\{\bar{V}_{\alpha}: x \in \bar{V}_{\alpha}\right\}$. Since $v$ is a locally finite collection, $V$ is closure preserving. Thus $U$ is an open set containing $x$ which intersects at most $n$ members of $v$.

THEOREM 4. Let $n$ be a positive integer and let $X$ be an n-boundedly metacompact collectionwise normal Hausdorff space. Then $X$ is $n$ boundedly paracompact.

Proof. The proof is similar to the proof of Theorem 3.

TheOREM 5 [1, TheORem 3.5]. Let $n$ be a positive integer and let $X$ be a paracompact Hausdorff space. Then $X$ has covering dimension $n-1$ if and only if $X$ is $n$-boundedly metacompact.

It might seem reasonable to conjecture that the product of a compact space and a boundedly paracompact space is boundedly paracompact or that an $F_{\sigma}$ subspace of a boundedly paracompact space is boundedly paracompact. These conjectures are shown to be false by Examples 5 and 6 respectively.

3. A characterization of bounded paracompactness in hereditarily paracompact spaces. Throughout this section all spaces are assumed to be Hausdorff.

Definition [2]. Let $X$ be a topological space and let $p \in X$. The dimension of $X$ at $p, \operatorname{dim}_{p} X$, is defined as follows: $\operatorname{dim}_{p} X$ is the least integer $n$ such that, for some open set $U$ containing $p, \operatorname{dim} \bar{U}=n$ or, if there is no such integer, $\operatorname{dim}_{p} X=\infty$.

Lemma 1. Let $X$ be a boundedly paracompact space and let $\left\{x_{n}\right\}_{n=1}^{\infty}$ be an infinite collection of distinct points of $X$ such that for each positive integer $n, \operatorname{dim}_{x_{n}} X \neq k$ for any $k \leqq n$. Then $\left\{x_{n}\right\}_{n=1}^{\infty}$ has a limit point.

Proof. Suppose $\left\{x_{n}\right\}_{n=1}^{\infty}$ has no limit point. Then there exists a discrete collection $\left\{U_{n}\right\}_{n=1}^{\infty}$ of open sets of $X$ such that for each $n$, $x_{n} \in U_{n}$. For each $n$, choose open sets $V_{n}$ and $W_{n}$ such that $x_{n} \in W_{n}$ $\subset \bar{W}_{n} \subset V_{n} \subset \bar{V}_{n} \subset U_{n}$. Since $\operatorname{dim}_{x_{n}} X \neq k$ for $k \leqq n, \bar{W}_{n}$ is not $k$-dimensional. By Theorem $5, \bar{W}_{n}$ is not $(n+1)$-boundedly metacompact, and hence it follows that $\bar{W}_{n}$ is not $(n+1)$-boundedly paracompact. Hence there is an open cover $\mathfrak{W}_{n}$ of $\bar{W}_{n}$ which has no locally finite open refinement of order $n+1$. Let $V_{n}=\left\{Z_{\alpha}: Z_{\alpha}\right.$ is open in $V_{n}$ and $\left.Z_{\alpha} \cap \bar{W}_{n} \in W_{n}\right\}$. Let $\mathfrak{U}_{n}$ be the open cover of $U_{n}$ consisting of $\mathcal{V}_{n}$ and the set $U_{n}-\bar{W}_{n}$. Let $\mathfrak{u}=\left(\cup_{n=1}^{\infty} \mathfrak{U}_{n}\right) \cup\left\{X-\operatorname{cl}\left(\cup_{n=1}^{\infty} V_{n}\right)\right\}$. Then $\mathfrak{U}$ is an open cover of $X$, since $\operatorname{cl}\left(\bigcup_{n=1}^{\infty} V_{n}\right)=\bigcup_{n=1}^{\infty} \bar{V}_{n}$.

Let $N$ be a positive integer and let $v$ be an open refinement of $u$. Let $\mathscr{W}_{N}^{*}=\left\{A \cap \bar{W}_{N}: A \in \mathcal{V}\right\}$. There exists $x \in \bar{W}_{N}$ such that each open 
set about $x$ intersects $N+2$ members of $w_{N}^{*}$. Hence each open set about $x$ intersects $N+2$ members of $v$. This contradicts the hypothesis that $X$ is boundedly paracompact.

Lemma 2. If $X$ is a boundedly paracompact space, then the set of points of $X$ at which $X$ is infinite-dimensional is compact.

Proof. Let $X$ be a boundedly paracompact space and let $A$ be the set of points at which $X$ is infinite-dimensional. Suppose $A$ is not compact. It is clear that $A$ is closed and hence metacompact. Thus there is an infinite collection $\left\{x_{n}\right\}_{n=1}^{\infty}$ of distinct points of $A$ which has no limit point in $A$. Since $A$ is closed, $\left\{x_{n}\right\}_{n=1}^{\infty}$ has no limit point in $X$. This contradicts Lemma 1 . Thus $A$ is compact.

THEOREM 6. If $X$ is boundedly paracompact, then precisely one of the following is true.

(i) $X$ is finite-dimensional.

(ii) $X$ is nonempty, compact, and infinite-dimensional at each of its points.

(iii) $X=A \cup B$; where $A$ is nonempty and compact, $X$ is infinitedimensional at each point of $A, B$ is nonempty and finite-dimensional, and $X$ is finite-dimensional at each point of $B$.

(iv) $X=A \cup\left(\cup_{n=0}^{\infty} A_{n}\right)$; where $A$ is nonempty and compact, and $X$ is infinite-dimensional at each point of $A ; A_{n}$ is nonempty for infinitely many $n$, and for each $n$ such that $A_{n} \neq \varnothing, X$ is $n$-dimensional at each point of $A_{n} ; \cup_{n=0}^{\infty} A_{n}$ is infinite-dimensional; and if $\left\{n_{i}\right\}_{i=1}^{\infty}$ is any subsequence of the nonnegative integers such that for each positive integer $i$, $x_{i} \in A_{n_{i}}$, then $\operatorname{cl}\left(\left\{x_{i}\right\}_{i=1}^{\infty}\right) \cap A \neq \varnothing$.

Proof. Let $X$ be a boundedly paracompact space which does not satisfy (i) or (ii). Let $A=\left\{a: \operatorname{dim}_{a} X=\infty\right\}$ and for each nonnegative integer $n$, let $A_{n}=\left\{x: \operatorname{dim}_{x} X=n\right\}$.

Suppose first that $A=\varnothing$. If only finitely many $A_{n}$ were nonempty, then by [2, Theorem 3.3], $X$ would be finite-dimensional. As this is not the case, there are infinitely many nonnegative integers $n$ for which $A_{n} \neq \varnothing$. Hence there is an infinite subset $\left\{x_{i}\right\}_{i=1}^{\infty}$ of distinct points of $X$ such that for each positive integer $i, \operatorname{dim}_{x_{i}} X \neq k$ for any positive integer $k \leqq i$. By Lemma $1,\left\{x_{i}\right\}_{i=1}^{\infty}$ has a limit point $x \in X$. It is easy to see that $x \in A$. Thus $A \neq \varnothing$, and by Lemma $2, A$ is compact.

Since $X$ does not satisfy (ii), there is a positive integer $p$ such that $A_{p} \neq \varnothing$. If $\bigcup_{n=0}^{\infty} A_{n}$ is finite-dimensional, then $X$ satisfies (iii) where $B$ is taken to be $\bigcup_{n=0}^{\infty} A_{n}$. Otherwise, $\bigcup_{n=0}^{\infty} A_{n}$ is infinite-dimensional 
and so for infinitely many $n, A_{n} \neq \varnothing$. Then by Lemma $1, X$ satisfies (iv).

It can be seen that the classes of spaces satisfying these four statements are mutually exclusive. Thus if $X$ is boundedly paracompact, it satisfies exactly one of the conditions of the theorem.

THEOREM 7. Let $X$ be a hereditarily paracompact space which satisfies one of the four statements of Theorem 6. Then $X$ is boundedly paracompact.

Proof. We shall continue to use the notation of Theorem 6. Clearly if $X$ satisfies either (i) or (ii), then $X$ is boundedly paracompact.

Suppose $X$ satisfies (iii) and let $\mathcal{u}=\left\{U_{\alpha}: \alpha \in Q\right\}$ be an open cover of $X$. Since $X$ is paracompact, there is a one-to-one open refinement $\mathfrak{F}=\left\{H_{\alpha}: \alpha \in Q\right\}$ of $\mathcal{u}$ such that $\bar{H}_{\alpha} \subset U_{\alpha}$ for each $\alpha \in Q$. Since $A$ is compact, there is a finite subcollection $\left\{H_{\alpha_{i}}: 1 \leqq i \leqq k\right\}$ of $\mathcal{F C}$ which covers $A$. Let $V=\{H \cap B: H \in \mathcal{H}\}$. Since $B$ is finite-dimensional and paracompact, $B$ is boundedly paracompact. Hence there is a positive integer $m$ and an open locally finite refinement $W$ of $v$ of order $m$. Let $N=X-\cup_{i=1}^{k} \bar{H}_{\alpha_{i}}$ and let ' $\mathcal{W}^{\prime}=\{W \cap N: W \in W\}$. Define $\mathcal{G}$ to be $W^{\prime} \cup\left\{U_{\alpha_{i}}: 1 \leqq i \leqq k\right\}$. Since $B$ is open in $X, g$ is an open locally finite refinement of $u$ of order less than or equal to $m+k$. Therefore $X$ is boundedly paracompact.

Suppose $X$ satisfies (iv). Let $\mathcal{u}=\left\{U_{\alpha}: \alpha \in Q\right\}$ be an open cover of $X$. There is a one-to-one open refinement $\mathcal{v}=\left\{V_{\alpha}: \alpha \in Q\right\}$ of $\mathcal{u}$ such that $\bar{V}_{\alpha} \subset U_{\alpha}$ for each $\alpha \in Q$. Since $A$ is compact, there is a finite subcollection $\mathcal{U}^{\prime}=\left\{V_{\alpha_{i}}: 1 \leqq i \leqq k\right\}$ which covers $A$. Suppose there are infinitely many $A_{n}$ which are not covered by $\mathcal{V}^{\prime}$. For each integer $n$ such that $A_{n}$ is not covered by $\mathcal{V}^{\prime}$, let $x_{n} \in A_{n}-U \mathcal{U}^{\prime}$. The set of all such $x_{n}$ has a limit point $x \in A$, and there exists $V \times \mathcal{V}^{\prime}$ such that $x \in V$. But then there is some $x_{n} \in V$. This contradicts the assumption that $x_{n} \in A_{n}-U V^{\prime}$. Thus there are only finitely many $A_{n}$ which are not covered by $\mho^{\prime}$. Let $m$ be the largest nonnegative integer such that $A_{m}$ is not covered by $\mathcal{U}^{\prime}$. Let $B=X-\bigcup_{i=1}^{m} \bar{V}_{\alpha_{i}} \subset \bigcup_{i=0}^{m} A_{i}$. Then $B$ is open in $X$ and for each $b \in B, \operatorname{dim}_{b} X \leqq m$. Thus for each $b \in B$, $\operatorname{dim}_{b} B \leqq m$. By [2, Theorem 3.3], $B$ is finite-dimensional. The remainder of the proof that $X$ is boundedly paracompact follows from an argument similar to the argument of the previous paragraph.

An alternate characterization of bounded paracompactness in hereditarily paracompact spaces is the following theorem.

THEOREM 8. If $X$ is a hereditarily paracompact Hausdorff space, then $X$ is boundedly paracompact if and only if $X=A \cup\left(\cup_{n=1}^{\infty} A_{n}\right)$, 
where $A$ is compact and $X$ is infinite-dimensional at each point of $A$, and where each $A_{n}$ is open in $X$ and finite-dimensional, such that every sequence in $\bigcup_{n=1}^{\infty} A_{n}$ having no limit point in $X$ is residual in some $A_{n}$.

Proof. Suppose that $X$ is boundedly paracompact. For each positive integer $n$, let $\mathcal{u}_{n}=\{U: U$ is an open subset of $X$ and $\operatorname{dim} \bar{U} \leqq n\}$ and let $A_{n}=\bigcup\left\{U: U \in \mathcal{U}_{n}\right\}$. Let $A=X-\bigcup_{n=1}^{\infty} A_{n}$. Let $n$ be a positive integer. Since $A_{n}$ is paracompact, $\mathfrak{u}_{n}$ has a closed locally finite refinement $\mathrm{g}$. Suppose that $G \in \mathcal{G}$. Since $G$ is closed and contained in some member $U$ of $\mathfrak{u}_{n}, \operatorname{dim} G \leqq \operatorname{dim} \bar{U} \leqq n$. Hence by the General Sum Theorem, $\operatorname{dim} A_{n} \leqq n$. Since $X$ is infinite-dimensional at each point of $A$, by Lemma 2, $A$ must be compact. Lemma 1 shows that every sequence in $\bigcup_{n=1}^{\infty} A_{n}$ which is not residual in any $A_{n}$ must have a limit point.

The proof of the converse can be shown in a manner similar to the proof of Theorem 7 .

Corollary. If $X$ is a metric space, then $X$ is weakly infinite-dimensional if and only if $X$ is boundedly paracompact and the set of points of $X$ at which $X$ is infinite-dimensional is weakly infinite-dimensional.

This follows from Theorem 8 and [5, Theorem VI.6].

4. Examples. We now summarize the relationship of the properties we have been investigating to other topological properties.

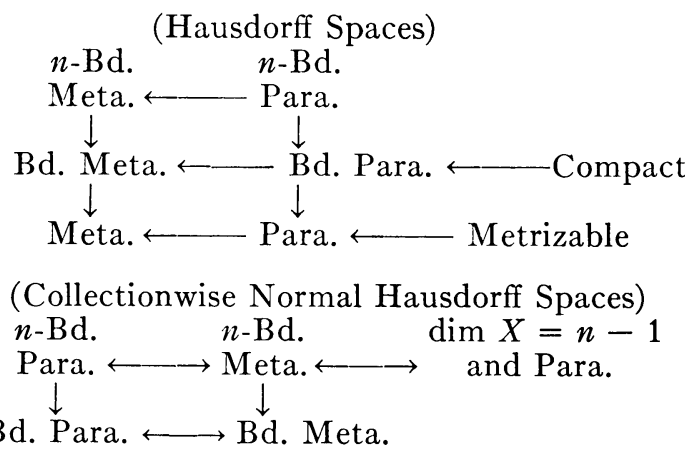

The following examples show that there are no additional implications among these properties.

EXAMPLE 1. A metrizable space which is not boundedly metacompact. By Theorems 3 and 6, any locally infinite-dimensional metric space which is not compact will suffice (e.g. Hilbert space).

EXAMPle 2. A 1-boundedly (0-dimensional) paracompact Hausdorff space which is neither compact nor metrizable. 
Let $(R, J)$ be the real line with the half-closed half-open interval topology.

Example 3. A 2-boundedly metacompact Hausdorff space which is not paracompact.

Let $R$ be the real line and let $A=\{1 / n: n$ is a positive integer $\}$. Let $\varepsilon$ be the usual Euclidean topology for $R$ and let $J=\{U-B: U \in \mathcal{E}$ and $B \subset A\}$. Then $(R, J)$ has the desired properties.

EXAmple 4. A metrizable (collectionwise normal) boundedly metacompact space which is not (finite-dimensional) n-boundedly metacompact for any positive integer $n$.

The Hilbert cube has the desired properties.

Example 5. A compact metric space $X$ and a boundedly paracompact metric space $Y$ such that $X \times Y$ is not boundedly paracompact.

Let $X$ be the Hilbert cube and let $Y$ be the real line. It follows from Theorem 6 that $X \times Y$ is not boundedly paracompact.

EXAmple 6. An $F_{\sigma}$-subspace of a boundedly paracompact Hausdorff space which is not boundedly paracompact. For each positive integer $n$, let $B_{n}$ be the closed standard $n$-ball in Euclidean $n$-space and imbed $B=\bigcup_{n=1}^{\infty} B_{n}$ as a subspace of the Hilbert cube so that for $i \neq j$, $B_{i} \cap B_{j}=\varnothing$. Then $B$ is an $F_{\sigma}$-subspace of the Hilbert cube, but $B$ is not boundedly paracompact.

\section{REFERENCES}

1. C. H. Dowker, Mapping theorems for non-compact spaces, Amer. J. Math. 69 (1947), 200-242. MR 8, 594.

2. - Local dimension of normal spaces, Quart. J. Math. Oxford Ser. (2) 6 (1955), 101-120. MR 19, 157. 1136.

3. J. L. Kelley, General topology, Van Nostrand, Princeton, N. J., 1955. MR 16,

4. E. Michael, A note on paracompact spaces, Proc. Amer. Math. Soc. 4 (1953), 831-838. MR 15, 144.

5. Jun-iti Nagata, Modern dimension theory, Bibliotheca Mathematica, vol. 6, Interscience, New York, 1965. MR 34 \#8380.

Virginia Polytechnic Institute, Blacksburg, Virginia 24061 